\title{
De novo variants in population constrained fetal brain enhancers and intellectual disability
}

Matias G De Vas, $\mathrm{PhD}^{1}$, Myles G Garstang, $\mathrm{PhD}^{2,3}$, Shweta S Joshi, MSc ${ }^{1}$, Tahir N Khan, $\mathrm{PhD}^{2,4}$, Goutham Atla, BE ${ }^{1,5,6}$, David Parry, $\mathrm{PhD}^{7}$, David Moore, $\mathrm{PhD}^{8}$, Ines Cebola, $\mathrm{PhD}^{1}$, Shuchen Zhang, $\mathrm{PhD}^{9}$, Wei Cui, $\mathrm{PhD}^{9}$, Anne K Lampe, $\mathrm{PhD}^{8}$, Wayne W Lam, $\mathrm{PhD}^{8}$, David R FitzPatrick, MD, $\mathrm{PhD}^{7}$, Jorge Ferrer, MD, $\mathrm{PhD}^{1,5,6}$, Madapura M Pradeepa, $\mathrm{PhD}^{2,3^{*}}$ and Santosh S Atanur, $\mathrm{PhD}^{1,10^{*}}$

1 - Section of Epigenomics and Disease, Department of Medicine, Imperial College London, London W12 0NN, UK

2 - Blizard institute, Barts and The London School of Medicine and Dentistry, Queen Mary University of London, London, E1 2AT

3 - School of Biological Sciences, University of Essex, Colchester, CO4 3SQ

4 - Department of Biological Sciences, National University of Medical Sciences (NUMS), Rawalpindi, Pakistan

5 - Bioinformatics and Genomics Program, Centre for Genomic Regulation (CRG), The Barcelona Institute of Science and Technology (BIST), 08003 Barcelona, Spain

6 - Centro de Investigación Biomédica en Red de Diabetes y Enfermedades Metabólicas Asociadas (CIBERDEM) Barcelona, Spain

7 - MRC Human Genetics Unit, University of Edinburgh, EH4 2XU

8 - South-East Scotland Regional Genetics Service, Western General Hospital, Edinburgh $\mathrm{EH} 42 \mathrm{XU}$

9 - Institute of Reproductive and Developmental Biology, Faculty of Medicine, Imperial College London, London, W12 0NN

10 - Previous institute: Centre for Genomic and Experimental Medicine, University of Edinburgh, Edinburgh EH4 2XU

* Corresponding authors

Correspondence to Santosh Atanur email: satanur@ic.ac.uk 
bioRxiv preprint doi: https://doi.org/10.1101/621029; this version posted September 8, 2019. The copyright holder for this preprint (which was not certified by peer review) is the author/funder. All rights reserved. No reuse allowed without permission.

or Madapura Pradeepa email: p.m.madapura@qmul.ac.uk 


\section{Abstract}

Purpose: The genetic aetiology of a major fraction of patients with intellectual disability (ID) remains unknown. De novo mutations (DNMs) in protein-coding genes explain up to $40 \%$ of cases, but the potential role of regulatory DNMs is still poorly understood.

Methods: We sequenced 70 whole genomes from 24 ID probands and their unaffected parents and analyzed 30 previously sequenced genomes from exomenegative ID probands.

Results: We found that DNVs were selectively enriched in fetal brain-specific enhancers that show purifying selection in human population. DNV containing enhancers were associated with genes that show preferential expression in the prefrontal cortex, have been previously implicated in ID or related disorders, and exhibit intolerance to loss of function variants. DNVs from ID probands preferentially disrupted putative binding sites of neuronal transcription factors, as compared to DNVs from healthy individuals and most showed allele-specific enhancer activity. In addition, we identified recurrently mutated enhancer clusters that regulate genes involved in nervous system development (CSMD1, OLFM1 and POU3F3). Moreover, CRISPR-based perturbation of a DNV-containing enhancer caused CSMD1 overexpression and abnormal expression of neurodevelopmental regulators.

Conclusion: Our results, therefore, provide new evidence to indicate that DNVs in constrained fetal brain-specific enhancers play a role in the etiology of ID.

Keywords: Genome sequencing, Intellectual Disability, Enhancers, Non-coding variants, Medical Genomics 
bioRxiv preprint doi: https://doi.org/10.1101/621029; this version posted September 8, 2019. The copyright holder for this preprint (which was not certified by peer review) is the author/funder. All rights reserved. No reuse allowed without permission.

\section{Conflict of Interest}

The authors declare no conflict of interest. 


\section{Introduction}

Intellectual disability (ID) is a neurodevelopmental disorder that is characterized by limitations in both intellectual functioning and adaptive behavior ${ }^{1}$. The clinical presentation of ID is heterogeneous, often coexisting with congenital malformations or other neurodevelopmental disorders such as epilepsy and autism ${ }^{1}$, and the worldwide prevalence is thought to be near $1 \%^{2}$. Throughout the past decade, great progress has been made in identifying the genetic causes of ID. De novo protein truncating variants and copy number variants affecting a large set of protein-coding genes explain up to $40 \%$ of ID cases ${ }^{1,3,4}$, whereas the genetic etiology of a major fraction of ID patients still remains unknown. De novo variants (DNV) in non proteincoding regions of the genome could plausibly explain some of the cases in which no causal coding variant has been identified.

Previous studies have implicated variants in long-range transcriptional regulatory elements, also known as enhancers, in monogenic developmental disorders including preaxial polydactyly $(S H H)^{5,6}$, Pierre Robin sequence $(S O X 9)^{7}$, congenital heart disease $(T B X 5)^{8}$ and pancreatic agenesis $(P T F 1 A)^{9}$. A systematic analysis of variants in evolutionarily ultra-conserved regions of the genome has estimated that around $1-3 \%$ of the developmental disorder patients without disease-causing coding variants would carry disease-causing DNVs in fetal brain-active regulatory elements $^{10}$. A large-scale genome sequencing of patients with autism spectrum disorder (ASD) demonstrate that DNVs in conserved promoter regions contribute to ASD, while no significant association was found between enhancer variants and $\mathrm{ASD}^{11}$.

Despite these precedents, the efforts to implicate enhancer variants in human disease, however, face numerous challenges. Importantly, it is currently not possible 
to readily discern functional enhancer variants from non-functional or neutral variants based on sequence features. This can be partially addressed through experimental analysis of regulatory DNA variants. Moreover, we still need a full understanding of which regulatory regions and which subsequences within the regulatory regions are most likely to harbor disruptive variants. In addition, one of the biggest challenges in interpreting variants in regulatory regions is to correctly associate regulatory regions to the potential target genes. Systematic identification of tissue specific promoterenhancer interaction maps would help identification of regulatory regions that are associated with disease relevant genes.

To address some of these challenges, we have sequenced genome of patients with ID, and examined whether DNVs target a subset of regulatory regions that are most likely to harbor etiological defects. We hypothesized that ID could result from DNVs in enhancers that are specifically active during brain development. We further reasoned that although evolutionary conservation is an important metric to prioritize genomic regions, advanced human cognition has been attributed to human fetal brain enhancers that are gained during evolutionary expansion and elaboration of the human cerebral cortex ${ }^{12}$, hence critical regulatory sequences for intellectual functions may show sequence constraints within human populations regardless of their evolutionary conservation.

In this study, we deployed whole genome sequence analysis, integrative genomic, epigenomic and experimental studies to show that DNVs in patients with ID are selectively enriched in fetal brain-specific enhancers and human brain gained enhancers that exhibit sequence constraint within human populations. We further show that such DNVs map to enhancers that are associated with known ID genes, genes that are intolerant to variants and genes specifically expressed in the prefrontal cortex. Furthermore, we identify three fetal brain-specific enhancers domains with recurrent DNVs, and provide experimental evidence that candidate variants alter 
bioRxiv preprint doi: https://doi.org/10.1101/621029; this version posted September 8, 2019. The copyright holder for this preprint (which was not certified by peer review) is the author/funder. All rights reserved. No reuse allowed without permission.

enhancer activity in neuronal cells. These results provide new level of evidence that supports a role of DNVs in neurodevelopmental enhancers in the aetiology of ID. 


\section{Materials and methods}

\section{Selection criteria of intellectual disability patients for this study and ethical approval}

The inclusion criteria for this study were that the affected individuals had a severe undiagnosed developmental or early onset pediatric neurological disorder and that samples were available from both unaffected parents. Written consent was obtained from each patient family using a UK multicenter research ethics approved research protocol (Scottish MREC 05/MRE00/74).

\section{Sequencing and quality control}

Genome sequencing was performed on the Illumina X10 at Edinburgh Genomics. Genomic DNA (gDNA) samples were evaluated for quantity and quality using an AATI, Fragment Analyzer and the DNF-487 Standard Sensitivity Genomic DNA Analysis Kit. Next Generation sequencing libraries were prepared using Illumina SeqLab specific TruSeq Nano High Throughput library preparation kits in conjunction with the Hamilton MicroLab STAR and Clarity LIMS X Edition. The gDNA samples were normalized to the concentration and volume required for the Illumina TruSeq Nano library preparation kits then sheared to a 450bp mean insert size using a Covaris LE220 focused-ultrasonicator. The inserts were ligated with blunt ended, Atailed, size selected, TruSeq adapters and enriched using 8 cycles of PCR amplification. The libraries were evaluated for mean peak size and quantity using the Caliper GX Touch with a HT DNA 1k/12K/HI SENS LabChip and HT DNA HI SENS Reagent Kit. The libraries were normalised to $5 \mathrm{nM}$ using the GX data and the actual concentration was established using a Roche LightCycler 480 and a Kapa Illumina Library Quantification kit and Standards. The libraries were normalised, denatured, and pooled in eights for clustering and sequencing using a Hamilton MicroLab STAR with Genologics Clarity LIMS X Edition. Libraries were clustered onto HiSeqX Flow 
cell v2.5 on cBot2s and the clustered flow cell was transferred to a HiSeqX for sequencing using a HiSeqX Ten Reagent kit v2.5.

\section{Alignment and variant calling}

The de-multiplexing was performed using bcl2fastq (2.17.1.14) allowing 1 mismatch when assigning reads to a barcodes. Adapters were trimmed during the demultiplexing process. Raw reads were aligned to the human reference genome (build GRCh38) using the Burrows-Wheeler Aligner (BWA) mem $(0.7 .13)^{13}$. The duplicated fragments were marked using samblaster $(0.1 .22)^{14}$. The local indel realignment and base quality recalibration was performed using Genome Analysis Toolkit (GATK; 3.4$0-g 7 e 26428)^{15-17}$. For each genome SNVs and indels were identified using GATK (3.4-0-g7e26428) HaplotypeCaller ${ }^{18}$ creating a gvcf file for each genome. The gvcf files of all the individuals from the same family were merged together and regenotyped using GATK GenotypeGVCFs producing single VCF file per family.

\section{Variant filtering}

Variant Quality Score Recalibration pipeline from GATK ${ }^{15-17}$ was used to filter out sequencing and data processing artifacts (potentially false positive SNV calls) from true SNV and indel calls. First step was to create a Gaussian mixture model by looking at the distribution of annotation values of each input variant call set that match with the HapMap 3 sites and Omni 2.5M SNP chip array polymorphic sites, using GATK VariantRecalibrator. Then, VariantRecalibrator applies this adaptive error model to both known and novel variants discovered in the call set of interest to evaluate the probability that each call is real. Next, variants were filtered using GATK ApplyRecalibration such that final variant call set contains all the variants with 0.99 or higher probability to be a true variant call.

\section{De novo variants (DNV) calling and filtering}


The de novo variants (DNVs) were called using GATK Genotype Refinement workflow. First, genotype posteriors were calculated using sample pedigree information and the allele accounts from 1000 genome sequence data as a prior. Next, the posterior probabilities were calculated at each variant site for each sample of the trio. Genotypes with genotype quality $(\mathrm{GQ})<20$ based on the posteriors are filtered out. All the sites at which both the parents genotype was homozygous reference (0/0) and child's genotype was heterozygous (0/1), with GQs >= 20 for each sample of the trio, were annotated as the high confidence DNVs. Only high confident DNVs that were novel or had minor allele frequency less than 0.0001 in 1000 genomes project were selected for further analysis.

Because, the majority of the publically available datasets including epigenomic datasets are mapped to human genome assembly version hg19, we lifted over all the DNV co-ordinates to hg19 using liftover package. All the variant co-ordinates presented in this paper are from hg19 human genome assembly.

\section{DNV annotations}

DNV annotations were performed using Annovar ${ }^{19}$. To access DNV location with respect to genes, refseq, ENSEMBL and USCS annotations were used. To determine allele frequencies, 1000 genome, dbSNP, Exac and GnomAD databases were used. To determine pathogenicity of coding DNVs, annotations were performed with CADD, DANN, EIGAN, FATHMM and GERP++ pathogenicity prediction scores. In addition, we determined whether any coding DNV has been reported in ClinVar database as a disease-causing variant.

\section{Structural variant detection and filtering}

To detect structural variants (SV), we used four complimentary SV callers: BreakDancer v1.3.6 ${ }^{20}$, Manta v1.5.0 ${ }^{21}$, CNVnator v0.3.3 $3^{22}$ and CNVkit v0.9.6 $6^{23}$. The BreakDancer and Manta use discordant paired end and split reads to detect 
deletions, insertions, inversions and translocations, while CNVnator and CNVkit detect copy number variations (deletions and duplications) based on read-depth information. The consensus SV calls were derived using MetaSV v0.4 ${ }^{24}$. The MetaSV is the integrative SV caller, which merges SV calls from multiple orthogonal SV callers to detect SV with high accuracy. We selected SVs that were called by at least two independent SV callers out of four.

To detect de-novo SV, we used SV2 v1.4.125. SV2 is a machine-learning algorithm for genotyping deletions and duplications from paired-end genome sequencing data. In de novo mode SV2 uses trio information to detect de novo SVs at high accuracy.

\section{Tissue specific enhancer annotations}

Roadmap Epigenomic Project ${ }^{26}$ chromHMM segmentations across 127 tissues and cell types were used to define brain-specific enhancers. We selected all genic (intronic) and intergenic enhancers ("6_EnhG and 7_Enh) from male (E081) and female fetal brain (E082). This was accomplished using genome-wide chromHMM chromatin state classification in rolling 200bp windows. All consecutive 200bp windows assigned as an enhancer in fetal brain were merged to obtain enhancer boundaries. A score was assigned to each enhancer based on the total number of 200bp windows covered by each enhancer. Next, for each fetal brain enhancer, we counted the number of $200 \mathrm{bp}$ segments assigned as an enhancer in the remaining 125 tissues and cell types. This provided enhancer scores across 127 tissues and cell types for all fetal brain enhancers. To identify fetal brain specific enhancers, $Z$ scores were calculated for each fetal brain enhancer using the enhancer scores. Z scores were calculated independently for male and female fetal brain enhancers. Independent $Z$ score cutoffs were used for both male and female fetal brain enhancers such that approximately $35 \%$ of enhancers were selected. To define open accessible chromatin regions within brain-specific enhancers, we intersected 
enhancers with DNAse-seq data from Roadmap Epigenomic Project ${ }^{26}$ from male (E081) and female fetal brain (E082) respectively. Next, the male and female fetal brain specific enhancers were merged together to get final set of 27,420 fetal brain specific enhancers. We used similar approach to identify tissue specific enhancers for selected fetal and adult non-brain tissues.

\section{Human gain enhancers}

Human gain enhancers published previously by Reilly et $\mathrm{al}^{12}$ were downloaded from Gene Expression Omnibus (GEO) using accession number GSE63649.

\section{De novo variants from healthy individuals}

We downloaded de novo variants identified in healthy individual in genomes of the Netherland (GoNL) study ${ }^{27}$ from GoNL website.

\section{Fetal brain-specific genes}

Roadmap Epigenomic Project ${ }^{26}$ gene expression (RNA-seq) data from 57 tissues was used to identify fetal brain-specific genes. We used female fetal brain gene expression data, as RNA-seq data was available only for female fetal brain. For each gene, $Z$ scores were calculated using RPKM values across 57 tissues. The genes with $\mathrm{Z}$ score greater than two were considered as the brain specific genes.

\section{De novo variant enrichment analysis}

The expected number of de novo variants (DNVs) in fetal brain specific enhancers and human gain enhancers was estimated using the previously defined framework for de novo variants ${ }^{28}$. The framework for the null variant model is based on trinucleotide context where the second base is mutated. Using this framework, the probability of variant for each enhancer was estimated based on the DNA sequence of the enhancer. Probability of variant of all the enhancers within the enhancer set 
(fetal brain specific enhancers and human gain enhancers) was summed to estimate the probability of variant for the entire enhancer set. The probability of variant for fetal brain specific enhancers and human gain enhancers was estimated separately. To estimate the expected number of DNVs, the probability of variant for each enhancer set was multiplied by the cohort size $(n=47)$. To estimate the significance of observed number of DNVs over expected number, Poisson distribution probabilities were invoked using $\mathrm{R}$ function ppois.

\section{Enrichment of recurrently mutated enhancer clusters}

The enhancer clusters were randomly shuffled 1000 times. For each iteration we estimated number of enhancer clusters with more than one variant. Then we counted the number of times more than or equal to two variants were observed in three or more enhancer clusters. This number was then divided by 1000 to calculate P-value.

\section{DNV effect on transcription factor binding}

The $\mathrm{R}$ bioconductor package motifbreak $\mathrm{R}^{29}$ was used to estimate the effect of DNV on transcription factor binding. The motifbreakR works with position probability matrices (PPM) for transcription factors (TF). MotifbreakR was run using three different TF databases: viz. homer, encodemotif and hocomoco. To avoid false TF binding site predictions, either with reference allele or with alternate allele, a stringent threshold of 0.95 was used for motif prediction. DNVs that create or disturb a strong base (position weight $>=0.95$ ) of the TF motif, as predicted by motifbreakR, were selected for further analysis.

\section{Prediction of target genes of enhancers}

Three different methods were used to predict the potential target genes of enhancers.

Chromosome conformation capture (Hi-C) comprehensively detects chromatin 
interactions in the nucleus; however, it is challenging to identify individual promoterenhancer interactions using $\mathrm{Hi}-\mathrm{C}$ due to the complexity of the data. In contrast, promoter capture $\mathrm{Hi}-\mathrm{C}(\mathrm{PCHi}-\mathrm{C})$ specifically identifies promoter-enhancer interactions as it uses sequence capture to enrich the interactions involving promoters of annotated genes ${ }^{30}$. The significant interactions between promoters and enhancers identified using $\mathrm{PCHi}-\mathrm{C}$ in neuronal progenitor cells ${ }^{31}$ were used to assign target genes to the DNV containing enhancers. The enhancers were overlapped with the PCHi-C HindlII fragments. If an overlap was found between an enhancer and the PCHi-C HindIII fragment, the significantly interacting regions (PCHi-C HindIII fragments representing promoters of the genes) of the PCHi-C HindIII fragment were extracted to assign genes to the enhancers.

For an enhancer to interact with a promoter, both promoter and enhancer need to be active in specific cells at a specific stage. To identify promoter-enhancer interactions, all the active promoters in fetal brain (as defined by chromHMM segmentation) were extracted. Promoter-enhancer interactions occur within topologically associated domains (TAD) hence, promoters that were located within the same TAD as that of a DNV containing enhancer were used for analysis.

For each enhancer and promoter, H3K27ac counts were extracted from all tissues for which H3K27ac data was available in the Roadmap Epigenomic Project ${ }^{26}$ ChIPseq dataset. For fetal brain, H3K27ac ChIP-seq data published by Reilly et al ${ }^{12}$ was used because H3K27ac ChIP-seq data was not available in Roadmap Epigenomic Project ChIP-seq dataset for fetal brain. The Spearman rank correlation coefficient (Spearman's rho) was calculated between each enhancer-promoter pair within the TAD using Scipy stats.spearmanr function from Python. The pervariant test was performed to identify the significance of the correlation. The counts were randomly shuffled, independently for enhancers and promoters, 1000 times to calculate an adjusted $P$ value. The interactions with an adjusted $P$ value less than 0.01 were 
considered as a significant interaction between the enhancer and promoter.

Finally, if any enhancer remained unassigned to a gene using these approaches, they were assigned to fetal brain expressed genes within the TAD. A gene with an expression level more than or equal to 1 TPM in the Roadmap Epigenomic Project fetal brain RNA-seq data was considered to be expressed in the fetal brain.

\section{Gene enrichment analysis}

To test if enhancer associated genes were enriched for genes previously implicated in neurodevelopmental disorders, three different gene sets were used. 1) Intellectual disability (ID) gene list published in the review by Vissers et al ${ }^{1}$ was downloaded from Nature website 2) We compiled all the genes implicated in neurodevelopmental disorders in Deciphering Developmental Disorder (DDD) project ${ }^{32}$. 3) All the genes implicated in autism spectrum disorder were downloaded from SFARI browser. Significance of enrichment was tested using hypergeometric test in R.

Gene ontology enrichment and tissue enrichment analysis was performed using webbased tool Enricher (http://amp.pharm.mssm.edu/Enrichr/).

Probability of loss of function intolerance (pLI) scores for each gene was downloaded from Exome Aggregation Consortium (ExAC) browser (http://exac.broadinstitute.org/). Significance of enrichment was tested using hypergeometric test in $\mathrm{R}$.

\section{Cell culture}

LUHMES cells were purchased from ATCC (CRL-2927). Cells were cultured as previously described (Scholz et al, JNC 2011). Briefly, cells were attached on precoated multi-well plates (50 ug/mL poly-L-ornithine and $1 \mathrm{ug} / \mathrm{mL}$ fibronectin - Sigma) and grown in proliferation medium: Advanced DMEM/F-12 plus N-2 Supplement (Thermo Fisher), $2 \mathrm{mM}$ L-glutamine (Sigma) and $40 \mathrm{ng} / \mathrm{mL}$ recombinant basic 
fibroblast growth factor (R\&D Systems). Cells were differentiated into neurons for 7 days using standard differentiation medium: Advanced DMEM/F-12 plus N-2 Supplement, $2 \mathrm{mM}$ L-glutamine, $1 \mathrm{mM}$ dibutyryl cAMP (Sigma), $1 \mathrm{ug} / \mathrm{mL}$ tetracycline (Sigma) and $2 \mathrm{ng} / \mathrm{mL}$ recombinant human GDNF (R\&D Systems).

Neuroblastoma cell line (SH-SY5Y) was maintained in DMEM/F12 media (Gibco), $1 \%$ penicillin-streptomycin, $10 \%$ fetal bovine serum and $2 \mathrm{mM} \mathrm{L-glutamine.}$

\section{Dual luciferase enhancer assays}

Enhancer and control regions (500-600 bp) were amplified from human genomic DNA from HEK293T cells using Q5 High-Fidelity Polymerase (NEB). Amplified fragments were cloned into pGL4.23 plasmid (Promega), which consists of a minimal promoter and the firefly luciferase reporter gene. These regions were mutagenized in order to introduce the de novo variants of interest using the Q5 Site-Directed Mutagenesis kit (NEB) using non overlapping primers. pGL4.23 plasmids containing putative enhancer DNA were sequence verified and transfected, together with a Renilla luciferase expressing vector (pRL-TK Promega) into SHSY-5Y cells using Lipofectamine 3000 (Invitrogen) following manufacturer's protocol. Firefly and Renilla luciferase activity was measured 24 hours after transfection using the DualLuciferase Reporter Assay System (Cat. number E1910, Promega) as per the manufacturer's instructions. Primers used to amplify genomic DNA and for mutagenesis are provided in Table S11.

\section{CRISPR interference lentivirus preparation and transduction}

To generate viral particles, $293 \mathrm{FT}$ cells were transfected with $3^{\text {rd }}$ generation lentiviral plasmids (pMDLg/pRRE, pRSV-Rev and pMD2.G; Addgene \#12251,12253 and 12259, respectively) along with a plasmid encoding KRAB-dCAS9 (Addgene 118155) using the PElpro reagent (Polyplus-Transfection). Culture medium was changed the day after transfection (day 1 ) and virus was collected on day 3. Lentiviral particles 
were concentrated using Lenti-X Concentrator (Takara). LUHMES cells were seeded in 12-well plates and immediately transduced with $30 \mu$ of virus concentrate. Culture medium was changed the following day and selection $(4.5 \mu \mathrm{g} / \mathrm{mL}$ blasticidin) was started 3 days after transduction and continued until control untransduced cells were completely dead. A list of oligonucloetides used for sgRNA cloning is provided in Table S12.

\section{Genomic DNA extraction}

Genomic DNA was extracted by a modified version of the salting-out method. Briefly, cells were lysed in Lysis Buffer (100 mM Tris- $\mathrm{HCl}$ pH 8.5; 5 mM EDTA; 200 mM $\mathrm{NaCl} ; 0.2 \% \mathrm{SDS}$ ) plus $4 \mathrm{U} / \mathrm{mL}$ of Proteinase $\mathrm{K}$ (Thermo Fisher) for at least 2 hours at $55^{\circ} \mathrm{C}$ with agitation. Then, $0.4 \mathrm{x}$ volumes of $5 \mathrm{M} \mathrm{NaCl}$ were added to the mixture and centrifuged at max. speed for $10 \mathrm{~min}$. DNA in the supernatant was precipitated with $1 \mathrm{x}$ volume of isopropanol. After centrifugation, the pellet was washed with $70 \%$ ethanol and air dried for half an hour. DNA was resuspended in water and incubated for at least one hour at $37^{\circ} \mathrm{C}$ with agitation.

\section{RNA isolation, cDNA synthesis and RT-qPCR}

RNA was extracted using the RNeasy Mini Kit (QIAGEN) and cDNA was produced with the Transcriptor First Strand cDNA Synthesis Kit (Roche). RT-qPCR reactions were performed with SYBR Green Master Mix (Thermo Fisher) and run on an Applied Biosystems QuantStudio 12K Flex Real-Time PCR machine. Relative gene expression values were calculated with the $-\Delta$ Ct method, using TATA-Box Binding Protein (TBP) as a house-keeping gene for normalization. Oligonucleotides used for qPCR are provided in Table S13.

\section{Statistical analysis}


All luciferase experiments and gene quantification using QPCR were done in biological triplicates. The significance level was calculated using two-tailed t-test.

\section{RNA-seq and data analysis}

RNA-seq libraries using RNA extracted from control LUHMES cells, LUHMES cells with CSMD1 enhancer CRISPRi, control differentiated neurons and CRISPRi differentiated neurons were generated in triplicate. RNA-seq libraries were sequenced on one lane of Illumina Hi-seq 4000 with $75 \mathrm{bp}$ paired end sequencing.

Sequencing data for RNA-Seq samples are adapter trimmed using Fastp and mapped against a human reference genome GRCh38 using splice aware aligner STAR v2.6. $1^{33}$ with GENCODE v29 gene annotations (https://www.gencodegenes.org/human/release_29.html). We generated raw counts per gene using the FeatureCounts tool $(\mathrm{v} 1.6 .3)^{34}$. The differential expression analysis was performed using DESeq2 v1.24.0 $0^{35}$. 


\section{Results}

\section{Genome sequencing and identification of de novo variants}

We performed genome sequencing (GS) of 70 individuals including 24 probands with severe intellectual disability (ID) and their unaffected parents at an average genomewide depth of $37 \mathrm{X}$ (Table S1). Our cohort includes 22 trios and one quad family with two affected probands. We identified on average 4.08 million genomic variants per individual that include 3.36 million single nucleotide variants (SNVs) and 0.72 million short indels (Table S1). We focused our analysis on de novo variants (DNVs), as it has been shown that DNVs contribute significantly to neurodevelopmental disorders $^{32,36}$. We identified a total of 1,261 DNVs in 21 trios after excluding one trio from the analysis due to an excessively high number of DNVs. An average of 60 high quality DNVs per proband were identified, which includes 55.2 SNVs and 4.8 indels per proband (Table S2). The number of DNVs identified in this study is similar to the number of DNVs identified per proband in previous GS studies on neurodevelopmental disorders ${ }^{4,11,37}$. It has been shown that de novo copy number variants $\left(\mathrm{CNV}\right.$ s) play a significant role in severe $\mathrm{ID}^{4}$. We identified a total of three de novo CNVs in our ID probands (Table S3).

\section{Protein-coding de novo variants and copy number variants}

The role of protein truncating variants in ID is well established. Hence, we first looked at DNVs located in protein-coding regions of the genome. A total of $23 \mathrm{DNV}$ s were located in protein-coding regions (average 1.1 DNVs per proband). Of the 23 coding variants, 15 were non-synonymous coding variants or protein truncating variants. In six ID probands, we identified various types of potentially disease-causing variants in the genes KAT6A, TUBA1A, KIF1A, NRXN1 and PNKP, all of them previously implicated in $\mathrm{ID}^{1,4}$. The variant in KAT6A resulted in a premature stop codon while genes TUBA1A and KIF1A showed non-synonymous coding variants, which have 
been reported as a likely disease-causing and disease-causing respectively in ClinVar ${ }^{38}$ (Table S4). One de novo CNV resulted in partial deletion of NRXN1, a known ID gene. A family with two affected siblings was analyzed for the presence of recessive variants. We identified a homozygous $17 \mathrm{bp}$ insertion in the gene $P N K P$ (Table S4) in both siblings. This insertion has been reported as disease-causing in ClinVar ${ }^{38}$. These findings confirmed the disease-causing role of DNVs in ID.

\section{ID de novo variants are preferentially located in constrained fetal brain}

\section{enhancers}

In our severe ID cohort, we did not identify disease-causing coding DNVs in 17 ID cases $(\sim 70 \%)$, and hence decided to investigate potentially disease-causing variants in disease-relevant enhancer regions. We further analysed 30 previously published severe ID samples in which no disease-causing protein-coding DNVs have been found using $\mathrm{GS}^{4}$, yielding a total of 47 exome-negative ID cases.

We hypothesized that DNVs in fetal brain-specific enhancers could perturb expression levels of genes that are essential for brain development, leading to ID. We therefore identified 27,420 fetal brain-specific enhancers using the data from Roadmap Epigenomics project ${ }^{26}$ (see Methods). The majority (76.52\%) of these fetal brain specific enhancers were found to be candidate cis-Regulatory Elements (CCREs) defined by ENCODE3 ${ }^{39}$. In addition, we analyzed 8,996 human brain gained enhancers that have been shown to be active during cerebral corticogenesis ${ }^{12}$. All the downstream analysis was performed on a combined set of fetal brain-specific enhancers and human fetal brain gained enhancers, which we refer as fetal brain enhancers throughout the manuscript.

A total of $83 \mathrm{DNVs}$ (an average of 1.77 DNVs per proband) were located within fetal brain enhancers. It has been shown that the DNVs from individuals with neurodevelopmental disorders have been enriched in fetal brain active conserved 
non-coding elements ${ }^{10}$. Hence, we investigated enrichment of observed number of DNVs over expected in our ID cohort in multiple fetal and adult tissue specific enhancers including fetal brain and adult brain subsections using previously defined framework for interpreting $D N V s^{28}$. As a control we used a set of randomly selected, sequence composition matched quiescent regions and the DNVs identified in healthy individuals in the Genome of Netherlands $(\mathrm{GoNL})^{27}$. After applying multiple test correction we did not find a significant enrichment for DNVs in any tissue specific enhancers tested (Figure 1a). However, fetal brain enhancers showed the largest excess of DNVs as compared to the expected number of variants (Figure 1a). Other tissues showed expected or fewer DNVs compared to the expected while adult brain specific enhancers showed a depletion of DNVs (Figure 1a). This result was consistent with the expectation that variants in enhancers that are active during fetal brain development play a role in ID, which is a severe early-onset neurodevelopmental phenotype, rather than variants in enhancers that are active in adult brain or other tissues.

Despite that DNVs in ID (but not from healthy individuals) showed greatest enrichment in fetal brain enhancers compared to enhancers from other tissues or control regions, this result was not significant. We next explored whether subset of fetal brain enhancers that are under population constrain show enrichment for DNVs from ID patients.

Although variants in enhancer regions can lead to severe developmental defects ${ }^{40}$, functional redundancy of enhancers of developmental genes reduces the likelihood of severe functional consequences of enhancer variants ${ }^{41}$. Thus, disease-causing variants should specifically occur in enhancers that are intolerant to variants within human populations. The recently developed context dependent tolerance score (CDTS) provides sequence constraints across human population in non-coding regions of the genome at $10 \mathrm{bp}$ resolution ${ }^{42}$. The human gain enhancers as well as 
fetal brain-specific enhancers showed significant enrichment $\left(P<2.2 \times 10^{-16}\right.$ and $P<$ $2.2 \times 10^{-16}$ respectively) for constrained genomic regions (CDTS <=30; Figure $1 \mathbf{b}$ ) when compared to sequence composition-matched control regions. This finding suggests that the putative fetal brain enhancers tend to be intolerant to variants within human populations. A total of 25 fetal brain enhancer DNVs were located within constrained regions (CDTS score $<=30$ ) of the genome. We found marginal enrichment of DNVs in constrained enhancers $(C D T S<=30)$ as compared to control quiescent regions $(P=0.072 ;$ Figure 1c). However, when we restricted the analysis to highly constrained regions (CDTS $<=20$ ), we found significant excess of DNVs $(P=0.027$; Figure 1c) in fetal brain enhancers as compared to control regions. The increased burden of DNVs in ID patients in enhancers that are intolerant to variants within the human population suggests that DNVs in population-constrained regions are more likely to be functional.

\section{DNV-containing enhancers were associated with ID-relevant genes}

We next investigated the hypothesis that DNVs are preferentially located in enhancers that are connected with genes that are plausible etiological mediators of ID. To identify potential target genes of fetal brain enhancers we used the following datasets in sequential order: promoter capture $\mathrm{Hi}-\mathrm{C}(\mathrm{PCHi}-\mathrm{C})$ data ${ }^{31}$ from neuronal progenitor cells (NPC); correlation of H3K27ac ChiP-seq signal at promoters and enhancers across multiple tissues; and promoter-enhancer correlation using chromHMM segmentation data. The closest fetal brain expressed gene was assigned as a target gene for $24 \%$ of the enhancers that remained unassigned after application of these approaches. For all approaches, we restricted our search space to brain topologically associated domains (TADs) ${ }^{43}$ as the majority of enhancerpromoter interactions happen within TADs ${ }^{44}$. 
a

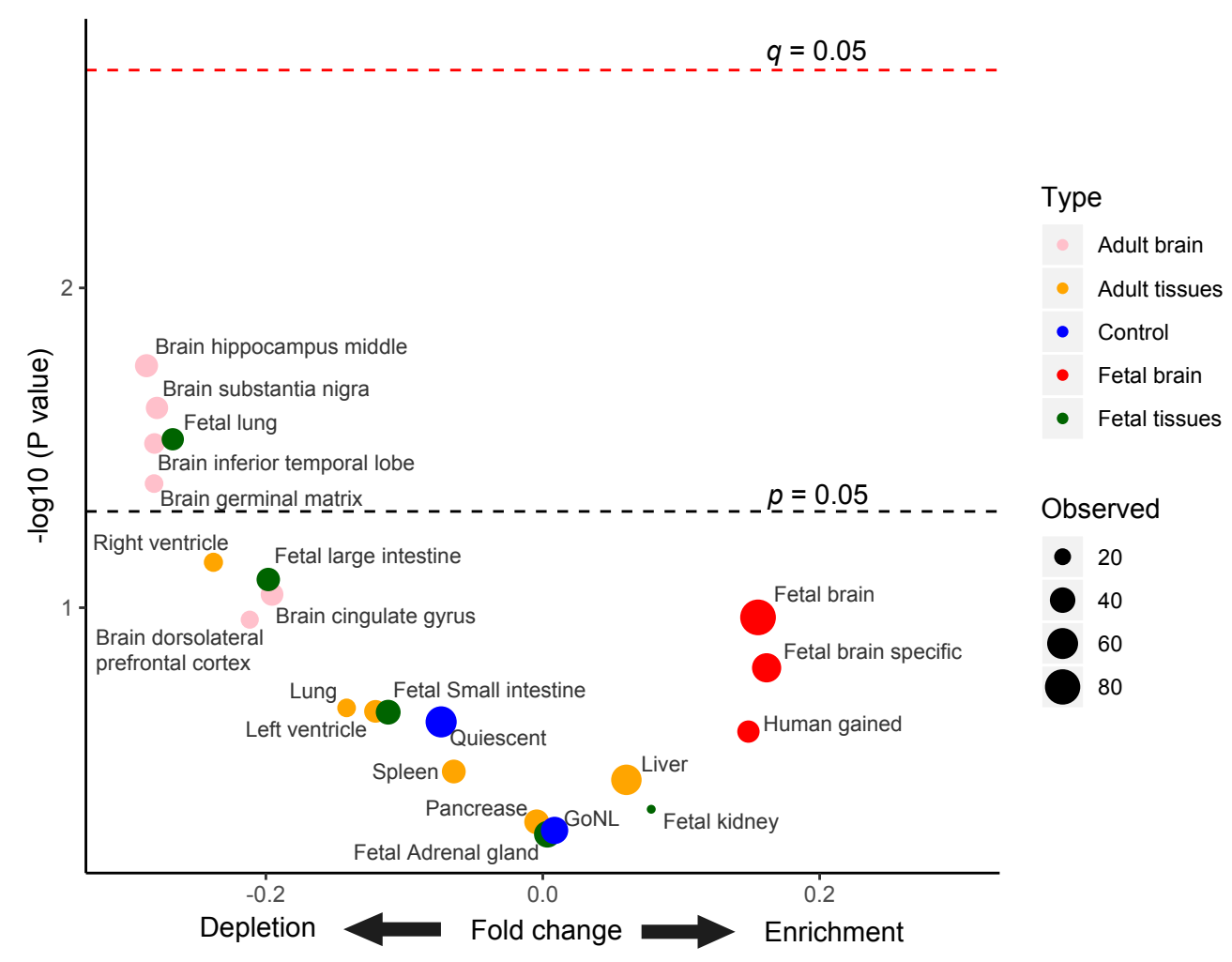

$b$

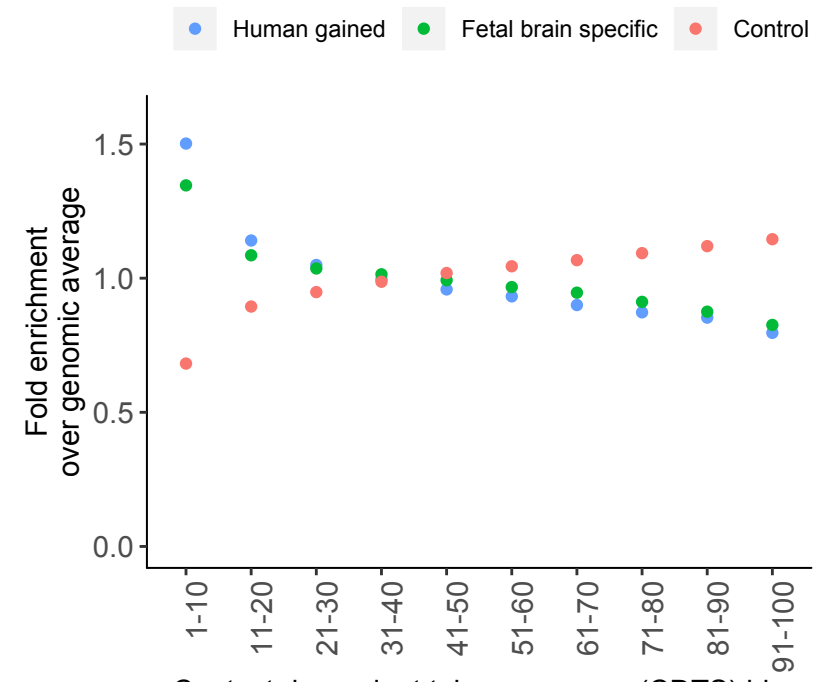

Context dependant tolerance score (CDTS) bin $c$

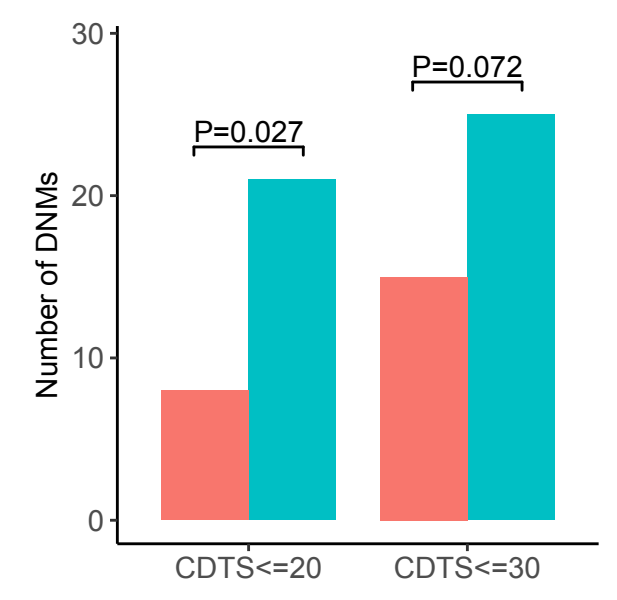

Figure 1: Enrichment of de novo variants (DNVs) in constrained enhancers. a) Enrichment or depletion of observed number of DNVs over expected number across multiple tissues and controls. The $\mathrm{x}$-axis scale is centred around zero which indicates an equal 
number of observed, and expected number, of DNVs. Positive numbers of $x$-axis indicate enrichment and negative numbers indicate depletion of DNVs. The red dots indicate fetal brain enhancers while pink dots indicate enhancers from adult brain subsections. Green dots indicate tissue specific enhancers from fetal tissues other than brain while orange dots indicate tissue specific enhancers from adult tissues. Two controls; sequence matched quiescent regions, and DNVs from healthy individuals from Genomes of The Netherland (GoNL) study overlapping fetal brain specific enhancers, are represented as blue dots. The size of the dot indicate observed number of DNVs. Black dotted line indicates $p$-value threshold of 0.05 while red dotted line indicate $p$-value after multiple test correction ( $q$-value). b) Enrichment of context dependent tolerance score (CDTS) across fetal brain specific and human gain enhancers. X-axis indicates bins of CDTS score. Y-axis indicates enrichment of CDTS relative to whole genome control. The blue dots indicate human gained enhancers; green dots indicate fetal brain specific enhancers while orange dots indicate sequence matched control regions. b) Number of de novo variants (DNVs) constrained enhancer (turquoise) and sequence matched control regions (orange).

Next, we compiled genes that have previously been implicated in ID or related neurodevelopmental disorders, using three gene sets; known ID genes ${ }^{4,45}$, genes implicated in neurodevelopmental disorders in the Deciphering Developmental Disorder (DDD) project $^{32}$ and autism risk genes (SFARI genes) ${ }^{46}$. This provided us with a unique set of 1,868 genes previously implicated in neurodevelopmental disorders. The genes that were connected with DNV-containing fetal brain enhancers were enriched for known neurodevelopmental disorder genes ( 25 genes, $P=0.025$;

\section{Table S5).}

We further observed that the target genes of DNV-containing enhancers were not only involved in nervous system development $\left(P=7.4 \times 10^{-4}\right.$; Table S6) but also predominantly expressed in the prefrontal cortex $\left(P=6.5 \times 10^{-3}\right.$; Table S7), a brain region that has been implicated in social and cognitive behavior, personality expression, and decision-making. 
The potential functional effect of heterozygous enhancer variants is expected to be mediated through altered expression of target genes. Recently, it has been shown that the majority of known severe haploinsufficient human disease genes are intolerant to loss of function (LoF) variants ${ }^{47}$. We compared the putative target genes of DNV-containing enhancers with the recently compiled list of genes that are intolerant to LoF variants $(\mathrm{pLI}>=0.9)^{47}$. We found that a significantly higher proportion of enhancer DNV target genes were intolerant to LoF variants than expected $\left(P=4.2 \times 10^{-5}\right.$; Table S8).

Taken together, our analysis shows that heterozygous DNVs are predominantly found in enhancers that are connected with genes that show preferential expression in the pre-frontal cortex, have been previously implicated in ID or related disorders, and exhibit intolerance to LoF variants.

\section{Recurrently mutated enhancer clusters}

We did not identify individual enhancers that are recurrently mutated (contains two or more DNVs from unrelated probands), but investigated whether clusters of enhancers that regulate the same gene show recurrent DNVs. We defined the clusters of enhancers as the set of enhancers that are connected with the same gene. The enhancer clusters associated with three genes, CSMD1, OLFM1 and POU3F3 were recurrently mutated with two DNVs in each of the enhancer clusters (Figure S1). A presence of three enhancer clusters with recurrent DNVs within the cohort of 47 ID probands was significantly higher than expected (pervariant test $P=$ 0.016). All three genes (CSMD1, OLFM1 and POU3F3) play a role in nervous system development ${ }^{48-50}$. Heterozygous variants in POU $3 F 3$ protein coding regions have been recently implicated in $I D^{51,52}$. A known role of these genes in nervous system development and the presence of recurrent variant in enhancer clusters 
associated with these genes in ID cohort suggest that these enhancer DNVs may contribute to ID.

\section{Functional disruption of enhancer function by ID DNVs}

Enhancers regulate gene expression through the binding of sequence-specific transcription factors (TFs) at specific recognition sites ${ }^{53}$. DNVs could elicit phenotypic changes because they alter the sequence of putative TF binding sites or because they create putative TF binding sites that have an impact on target gene expression. We used stringent criteria for TF motif prediction as well as motif disruption (see Methods). Of the 82 de novo SNVs that were located in fetal brain enhancers, 32 (39\%) were predicted to alter putative TFBS affinity, either by destroying or creating TFBS (Table S9a). In comparison only $23.5 \%$ of the DNVs from healthy individuals (GoNL) located in fetal brain enhancers were predicted to alter putative TFBS affinity (Table S9b). Thus, a significantly higher proportion of the fetal brain enhancer DNVs from ID probands found to disturb putative TFBS affinity as compared to GoNL DNVs (Fisher's exact test $p$-value $=0.0067$; Table S9c). Furthermore, the fetal brain enhancer DNVs from ID probands frequently disturbed putative binding sites of TFs that were predominantly expressed in neuronal cells $(P$ $=0.022$; Table S9d), whereas no such enrichment was observed for GoNL DNVs (Table S9e). Taken together our results suggest that the enhancer DNVs from ID probands were more likely to affect the binding sites of neuronal transcription factors, and could influence the regulation of genes involved in nervous system development through this mechanism.

To test the functional impact of regulatory variants on enhancer activity, we selected 11 potential disease-causing enhancer DNVs (Table S10), and investigated their functional impact in luciferase reporter assays in the neuroblastoma cell line $\mathrm{SH}$ SY5Y. Of the 11 enhancers containing DNVs, 10 showed significantly higher activity 
than the negative control in at least one allelic version, indicating that they do indeed function as active enhancers in this neuronal cell line (Figure 2). Amongst these 10 active enhancers, nine showed allele-specific activity, with five showing loss of activity and four showing gain of activity of the DNVs (Figure 2). Eight out of nine DNVs that showed allele-specific enhancer activity altered a core base of the TF motif with position specific weight $>=0.95$, and thereby disrupted the predicted affinity of the cognate TF (Table S9). The CSMD1 enhancer cluster had two DNVs (chr8: 2177122C>T and chr8: 2411360T>C) in two unrelated ID probands (Family 6 and Family 3 respectively). Both of these showed gain of activity compared to the wild type allele (Figure 2). By contrast, two DNVs in the OLFM1 enhancer cluster (chr9:137722838T>G and chr9:137333926C>T) from two unrelated ID probands (Family 4 and Family 12 respectively) caused loss of activity (Figure 2). These results demonstrate that selected DNVs from ID patients in fetal brain enhancers alter predicted TF binding affinity and have a functional impact on enhancer activity assays.

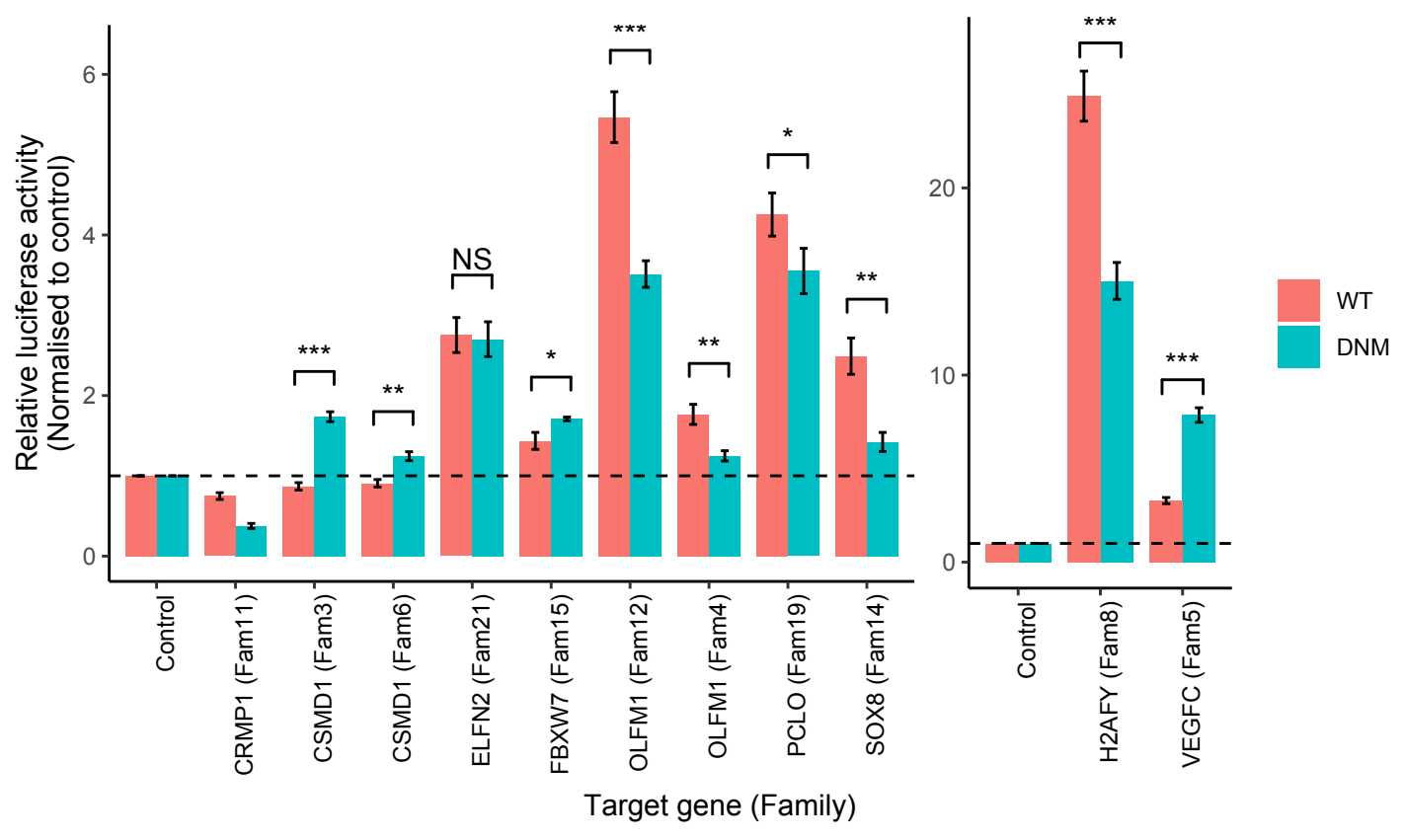


Figure 2: Effect of DNV on enhancer activity. Duel luciferase reporter assay of wild type (reference) and the mutant (DNV) allele. X-axis indicates putative target genes of the enhancer, while the family IDs are shown in brackets. Y-axis indicates relative luciferase activity normalised to empty plasmid. The error bars indicate standard error of means of three biological replicates. The enhancers associated with genes H2AFY and VEGFC are plotted with separately with different $Y$-axis scale because of high activity of these enhancers. The significance level was calculated using two-tailed t-test. ${ }^{* * *}$ Indicates $p$-value $<=0.001,{ }^{* *}$ indicate $p$-value between 0.01 and 0,001 while * indicates p-value between 0.01 to 0.05 .

\section{Function of the CSMD1 enhancer in a neural differentiation model}

We further explored the possible functional relevance of DNVs in the CSMD1 enhancer cluster. Both ID probands with these variants showed developmental delay and both were overgrown with high birth weights (above the 91st centile) and remain large throughout postnatal life. The CSMD1 gene is involved in neurogenesis ${ }^{54}$, and intronic variants have been shown to be associated with schizophrenia ${ }^{55}$. We focused on DNV chr8:2411360T>C from Family 3, because motif analysis predicted that it disrupts the binding site for the transcriptional repressor TCF7L1 ${ }^{56,57}$ (Figure 3a), which is known to inhibit premature neurogenesis ${ }^{58}$. The enhancer that harbors this DNV is located $2.4 \mathrm{Mb}$ away from the CSMD1 promoter (Figure 3a). To test the function of this enhancer, we performed lentiviral CRISPR interference (CRISPRi) by recruiting dCas9 fused with the repressor KRAB domain in the Lund human mesencephalic (LUHMES) neuronal precursor cell line, which do not express CSMD1 mRNA (Figure 3d), and then differentiated LUHMES cells into neurons. We found that CRISPRi of the CSMD1 enhancer led to significantly higher expression of CSMD1 in neurons than in non-targeted control cells ( $P=0.004$; Figure $3 \mathbf{b})$. Given that the KRAB domain represses transcription through heterochromatin spreading ${ }^{59,60}$ or simply by steric interference of endogenous regulatory components ${ }^{61}$, this result suggests that CRISPRi of this enhancer prevented binding by a transcriptional repressor, and thereby enabled overexpression of CSMD1. 
a

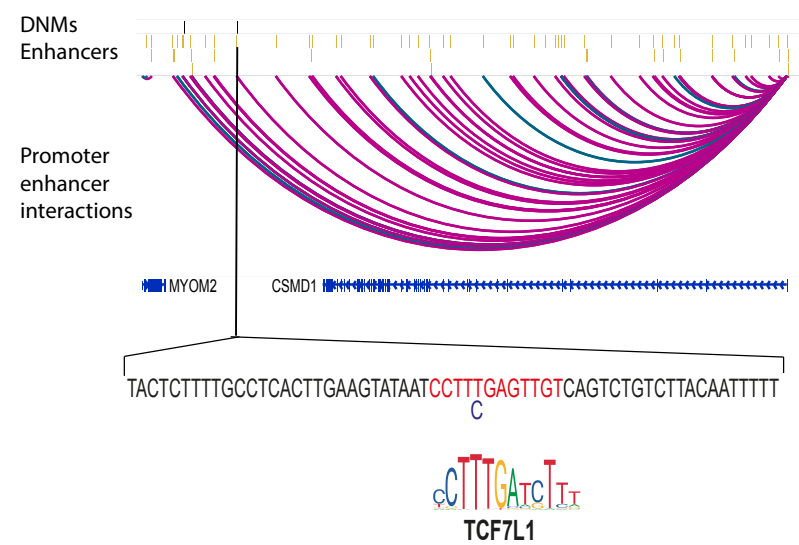

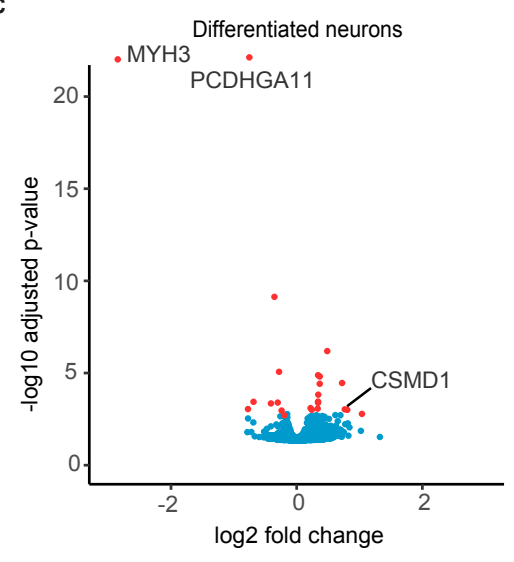

b

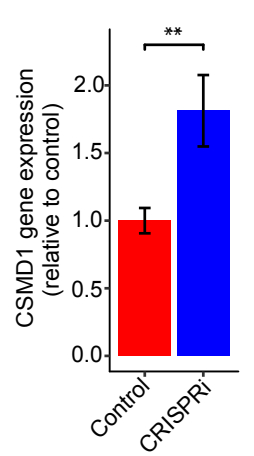

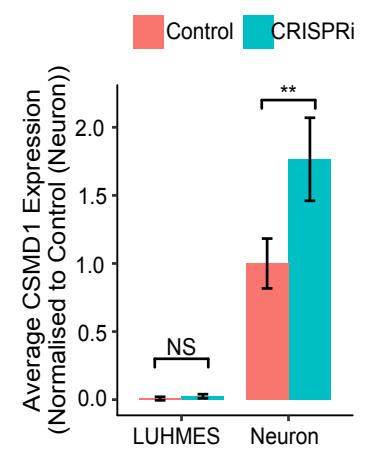

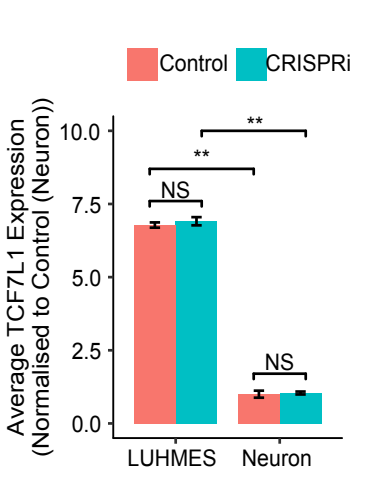

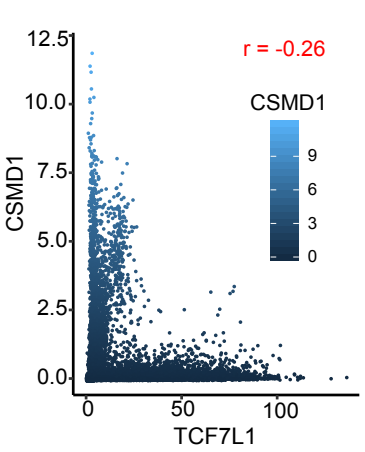

Figure 3: Do novo variant in CSMD1 enhancer lead premature activation of

CSMD1. a) Enhancer-promoter interactions in CSMD1 enhancer cluster. Pink arcs represent fetal brain specific enhancer CSMD1 promoter interactions while green arcs represent human gain enhancer-promoter interactions. De novo variant in CSMD1 enhancer $(T>C)$ alters the core base of the TCF7L1 transcription factor binding site. b) CSMD1 expression measured using qPCR. CSMD1 shows significantly higher expression in cells containing KRAB-dCas9 (blue bar) as compared to control wild type cells (red bars). The error bars indicate the standard error of means of three replicates. The significance level was calculated using a two-tailed t-test. c) Volcano plot depicting differentially expressed genes between CSMD1 enhancer CRISPRi and the control in differentiated neuron using RNA-seq. The red dots indicate significantly differentially expressed genes. d) Gene expression of CSMD1 and TCF7L1 genes in LUHMES cells and differentiated neurons, as determined by RNA-seq. e) Dot plot indicating gene expression pattern of CSMD1 and TCF7L1 across multiple tissues. Gene expression data was obtained from GTEx database. 
Because the DNV disrupts a high-affinity binding sequence for TCF7L1, the CRISPRi experiments suggest that TCF7L1 could be a negative regulator of CSMD1 during neuronal differentiation. Consistent with this model, TCF7L1 mRNA was highly expressed in LUHMES cells, which do not express CSMD1, and was downregulated upon differentiation to neurons, which do express CSMD1 (Figure 3d). Further analysis of GTEx ${ }^{62}$ showed that the expression of CSMD1 and TCF7L1 are almost mutually exclusive across human tissues (Figure 3e). Taken together, these results indicate that the candidate causal DNV disrupts a TCF7L1 recognition sequence in an enhancer that acts as a negative long range regulator of CSMD1 during neural differentiation.

To investigate the impact of perturbing the CSMD1 enhancer on neural differentiation, we performed RNAseq upon differentiation of neuronal precursors to neurons. RNAseq data confirmed significant up-regulation of CSMD1 in CRISPRi inactivated neurons as compared to controls. In addition, the genes MYH3 (myosin heavy chain 3 ), expressed exclusively during embryonic development ${ }^{63}$, and PCDHGA11 (Protocadherin Gamma-A11), which plays a significant role in establishment of cell-cell connections in the brain ${ }^{64}$ ac, showed a significant strong downregulation (Figure 3c). These experiments indicate that the enhancer that harbors the regulatory chr8:2411360T>C variant modulates critical regulators of axon formation and neuronal connectivity.

\section{CSMD1 enhancer cluster DNVs and ASD.}

The genetic underpinnings of both ID and autism spectrum disorders (ASD) have been ascribed to abnormal neuronal development, and may share molecular mechanisms ${ }^{65,66}$. Approximately $10 \%$ of individuals with ID have ASD, while $70 \%$ of individuals with ASD have some level of $I^{66}$. Therefore, we explored the occurrence of DNVs in the CSMD1 enhancer cluster in large-scale GS studies on ASD. The 
Simons Simplex Collection (SSC) has sequenced GS of 1,902 ASD families

(quads) $^{11}$, while the MSSNG database has compiled GS of 2,281 ASD trios ${ }^{37}$. Within the SSC cohort, four DNVs from four unrelated ASD cases were located in the CSMD1 enhancer cluster, while no DNVs in the CSMD1 enhancer cluster were found in 1,902 unaffected siblings. Additionally, we found four ASD patients from the MSSNG database harboring DNVs in the CSMD1 enhancer cluster. Thus, we identified a total of eight DNVs in ASD cohorts. Recurrent variants in the CSMD1 enhancer cluster in ID and ASD, but not in unaffected siblings, further reinforce the possible role of CSMD1 enhancer variants in these neurodevelopmental disorders. 


\section{Discussion}

Despite the recent widespread use of genome sequencing, the true burden of disease-causing variants in enhancers is unknown. This is largely due to an inability to predict the pathogenicity of enhancer variants based on sequence features. Aggregation of a minority of disease-causing variants with the majority of benign regulatory variants nullifies any signal from disease-causing variants in non proteincoding genomic regions in disease cohorts. It is noteworthy that in protein coding regions of the genome only protein-truncating variants, but not other protein-coding variants, show significant enrichment in neurodevelopmental disorders ${ }^{11,67}$. The analysis of DNVs in selected monogenic phenotypes provides a powerful analysis instrument, because it can focus on a relatively small number of variants that have increased likelihood of being disease-causing. In this study, we show that DNVs in a cohort of patients with ID exhibit a non-random genomic distribution that differs from DNVs observed in healthy individuals, with several features that are consistent with a disease-causing role of noncoding DNVs. DNVs from patients with ID were thus selectively enriched in fetal brain enhancers that exhibit variantal constraints in humans, in enhancers associated with genes that are ID-relevant, intolerant to loss of function variants and specifically expressed in pre-frontal cortex, and in diseaserelevant transcription factor binding sites. Our studies further provide experimental evidence indicating that ID DNVs are enriched for regulatory variants.

Nearly half of all human enhancers have evolved recently ${ }^{68}$, and advanced human cognition has been attributed to recently evolved human specific brain enhancers ${ }^{12}$. We show that fetal brain enhancers show selective constraint in humans (Figure 1b), and also found that DNVs in patients with ID are significantly enriched in fetal brain enhancers that are constrained in human populations. Enrichment of DNVs in enhancers that are intolerant to variants within human populations suggests that the DNVs in such essential enhancers are more likely to be functional. Consistent with 
this prediction, we show that DNVs from ID patients disturb binding sites of neuronal transcription factors within fetal brain enhancers more frequently than DNVs from healthy individuals. Furthermore, more than $90 \%$ of the DNVs tested showed allelespecific activity (Figure 2). Genetic and experimental observations, therefore, indicate that DNVs from patients with ID are enriched in functional neural regulatory variants.

The identification of genes that are recurrently mutated across multiple disease individuals has been a major route to discover novel disease genes ${ }^{69}$. We identified recurrent variants within three fetal brain enhancer clusters associated with genes involved in nervous system development (CSMD1, OLFM1, POU3F3), and found that this enrichment was significant relative to expectations. All three genes show high $\mathrm{pLI}$ score $(1,1,0.88$ respectively), indicating that they are intolerant to loss of function variants and dosage sensitive, and one of them (POU3F3) was recently shown to harbor disease-causing heterozygous variants in patients with $\mathrm{ID}^{52}$. Our results suggest that enhancer variants could lead to dysregulation of these genes during nervous system development and thereby contribute to the etiology of ID.

Among these three loci, we focused on CSMD1, a gene that is highly expressed in the central nervous system, particularly in the nerve growth cone ${ }^{50}$. Common genomic variants in CSMD1 are associated with schizophrenia and neuropsychological measures of general cognitive ability and memory function ${ }^{70,71}$, 72. Furthermore, CSMD1 knockout mice show strong neuropsychological defects ${ }^{73}$. Our motif sequence analysis, luciferase assays, and CRISPRi experiments indicate that an ID DNV in this locus affects an enhancer that acts as a negative regulator of CSMD1, and impacts expression of other neuronal genes. We further identified enrichment of variants in the CSMD1 enhancer cluster in cohorts of patients with neurodevelopmental disorders that have been proposed to share pathogenic 
mechanisms. Collectively, these findings implicate CSMD1 as a strong candidate target gene for ID disease-causing regulatory variants.

Taken together, our work has integrated whole genome sequences, epigenomics and functional analysis to examine the role of regulatory DNVs in ID. Despite the genetic heterogeneity of ID, which severely hampers efforts to unequivocal demonstrate a causal role for individual noncoding variants, our results provides multiple lines of evidence to indicate that functional regulatory variants in selectively constrained, stage-specific brain enhancers contribute to the etiology of ID. This work should prompt extensive genetic analyses and variant-specific experimental modeling to elucidate the precise role of regulatory variants in ID. 


\section{Acknowledgments}

We thank the families of the affected children for their time and support for the research. This research was supported by the National Institute for Health Research (NIHR) Imperial Biomedical Research Centre. This work was funded by grants from the Wellcome Trust Institute Strategic Support and National Institute for Health Research (NIHR) Imperial Biomedical Research Centre, Institute for Translational Medicine and Therapeutics (P70888) obtained by S.S.A.. J.F.'s work was funded by grants from the Wellcome Trust (WT101033 to J.F.), Medical Research Council (MR/L02036X/1 to J.F.), European Research Council Advanced Grant (789055 to J.F.). M.M.P.'s work was funded by the Wellcome Trust Research seed award and funding from the School of Medicine and Dentistry, QMUL. T.N.K. was partially supported by Government of Pakistan under PSDP project "Development of National University of Medical Sciences (NUMS), Rawalpindi". We thank Prof Andrew Jackson for helpful discussions and obtaining ethical approval for the study. We thank Mrs Sophie Shi for contributing to reagent generation. We also thank $\mathrm{Dr}$ Patrick Short and Dr Kaitlin Samocha both from Sanger Institute for providing trinucleotide probability table and helpful discussion on mutational model, respectively.

\section{Author contributions}

Conceptualization, S.S.A.; Acquiring funding, S.S.A.; Patient recruitment and sample collection, A.K.L, W.W.L. and D.R.F.; Bioinformatics analysis -Variant calling, S.S.J. and D.P.; Bioinformatics analysis: Enhancer-promoter interactions, G.A. and S.S.A.; Bioinformatics analysis: All other: S.S.A.; Experiments - Sanger sequencing: D.M.; Experiments - Luciferase: M.G.G., T.K., M.G.D., I.C., S.Z. and W.C.; Experiments CRISPR: M.G.D.; Writing - Original Draft: S.S.A.; Writing - Review \& Editing, J. F., M.M.P., M.G.D., I.C. and M.G.G.; Supervision, D.R.F., J.F., M.M.P. and S.S.A. 


\section{Data availability}

The whole genome sequence data as well as variant calls that support the findings of this study are available on request from the corresponding author [S.S.A.]. The data are not publicly available because it contains information that could compromise research participant privacy/consent. The RAN-seq data has been deposited to ArrayExpress with accession number E-MTAB-8027. RNA-seq data can be accessed using following URL https://www.ebi.ac.uk/arrayexpress/experiments/E-MTAB-8027/ 


\section{Reference}

1. Vissers, L. E. L. M., Gilissen, C. \& Veltman, J. A. Genetic studies in intellectual disability and related disorders. Nat. Rev. Genet. 17, 9-18 (2015).

2. Maulik, P. K., Mascarenhas, M. N., Mathers, C. D., Dua, T. \& Saxena, S. Prevalence of intellectual disability: A meta-analysis of population-based studies. Res. Dev. Disabil. 32, 419-436 (2011).

3. Deciphering Developmental Disorders Study. Prevalence and architecture of de novo mutations in developmental disorders. Nature 542, 433-438 (2017).

4. Gilissen, C. et al. Genome sequencing identifies major causes of severe intellectual disability. Nature 511, 344-347 (2014).

5. Lettice, L. A. et al. A long-range Shh enhancer regulates expression in the developing limb and fin and is associated with preaxial polydactyly. Hum. Mol. Genet. 12, 1725-1735 (2003).

6. Lettice, L. A., Devenney, P., De Angelis, C. \& Hill, R. E. The Conserved Sonic Hedgehog Limb Enhancer Consists of Discrete Functional Elements that Regulate Precise Spatial Expression. Cell Rep. 20, 1396-1408 (2017).

7. Benko, S. et al. Highly conserved non-coding elements on either side of SOX9 associated with Pierre Robin sequence. Nat. Genet. 41, 359-364 (2009).

8. Smemo, S. et al. Regulatory variation in a TBX5 enhancer leads to isolated congenital heart disease. Hum. Mol. Genet. 21, 3255-3263 (2012).

9. Weedon, M. N. et al. Recessive mutations in a distal PTF1A enhancer cause isolated pancreatic agenesis. Nat. Genet. 46, 61-64 (2014).

10. Short, P. J. et al. De novo mutations in regulatory elements in neurodevelopmental disorders. Nature 555, 611-616 (2018).

11. An, J.-Y. et al. Genome-wide de novo risk score implicates promoter variation in autism spectrum disorder. Science $\mathbf{3 6 2}$, eaat6576 (2018).

12. Reilly, S. K. et al. Evolutionary changes in promoter and enhancer activity during human corticogenesis. Science (80-. ). 347, (2015).

13. Li, H. \& Durbin, R. Fast and accurate short read alignment with BurrowsWheeler transform. Bioinformatics 25, 1754-1760 (2009).

14. Faust, G. G. \& Hall, I. M. SAMBLASTER: fast duplicate marking and structural variant read extraction. Bioinformatics 30, 2503-5 (2014). 
15. McKenna, A. et al. The Genome Analysis Toolkit: a MapReduce framework for analyzing next-generation DNA sequencing data. Genome Res. 20, 1297-303 (2010).

16. DePristo, M. A. et al. A framework for variation discovery and genotyping using next-generation DNA sequencing data. Nat. Genet. 43, 491-498 (2011).

17. Van der Auwera, G. A. et al. From FastQ Data to High-Confidence Variant Calls: The Genome Analysis Toolkit Best Practices Pipeline. in Current Protocols in Bioinformatics 11.10.1-11.10.33 (John Wiley \& Sons, Inc., 2013). doi:10.1002/0471250953.bi1110s43

18. Poplin, R. et al. Scaling accurate genetic variant discovery to tens of thousands of samples. bioRxiv 201178 (2017). doi:10.1101/201178

19. Wang, K., Li, M. \& Hakonarson, H. ANNOVAR: functional annotation of genetic variants from high-throughput sequencing data. Nucleic Acids Res. 38, e164-e164 (2010).

20. Chen, K. et al. BreakDancer: an algorithm for high-resolution mapping of genomic structural variation. Nat. Methods 6, 677-81 (2009).

21. Chen, X. et al. Manta: rapid detection of structural variants and indels for germline and cancer sequencing applications. Bioinformatics 32, 1220-1222 (2016).

22. Abyzov, A., Urban, A. E., Snyder, M. \& Gerstein, M. CNVnator: An approach to discover, genotype, and characterize typical and atypical CNVs from family and population genome sequencing. Genome Res. 21, 974-984 (2011).

23. Talevich, E., Shain, A. H., Botton, T. \& Bastian, B. C. CNVkit: Genome-Wide Copy Number Detection and Visualization from Targeted DNA Sequencing. PLOS Comput. Biol. 12, e1004873 (2016).

24. Mohiyuddin, M. et al. MetaSV: an accurate and integrative structural-variant caller for next generation sequencing. Bioinformatics 31, 2741-4 (2015).

25. Antaki, D., Brandler, W. M. \& Sebat, J. SV2: accurate structural variation genotyping and de novo mutation detection from whole genomes.

Bioinformatics 34, 1774-1777 (2018).

26. Kundaje, A. et al. Integrative analysis of 111 reference human epigenomes. Nature 518, 317-330 (2015).

27. Francioli, L. C. et al. Whole-genome sequence variation, population structure 
and demographic history of the Dutch population. Nat. Genet. 46, 818-825 (2014).

28. Samocha, K. E. et al. A framework for the interpretation of de novo mutation in human disease. Nat. Genet. 46, 944-950 (2014).

29. Coetzee, S. G., Coetzee, G. A. \& Hazelett, D. J. motifbreakR: an $\mathrm{R} /$ Bioconductor package for predicting variant effects at transcription factor binding sites: Fig. 1. Bioinformatics 31, btv470 (2015).

30. Javierre, B. M. et al. Lineage-Specific Genome Architecture Links Enhancers and Non-coding Disease Variants to Target Gene Promoters. Cell 167, 13691384.e19 (2016).

31. Freire-Pritchett, P. et al. Global reorganisation of cis-regulatory units upon lineage commitment of human embryonic stem cells. Elife 6, e21926 (2017).

32. McRae, J. F. et al. Prevalence and architecture of de novo mutations in developmental disorders. Nature 542, 433-438 (2017).

33. Dobin, A. et al. STAR: ultrafast universal RNA-seq aligner. Bioinformatics 29, 15-21 (2013).

34. Liao, Y., Smyth, G. K. \& Shi, W. The Subread aligner: fast, accurate and scalable read mapping by seed-and-vote. Nucleic Acids Res. 41, e108 (2013).

35. Love, M. I., Huber, W. \& Anders, S. Moderated estimation of fold change and dispersion for RNA-seq data with DESeq2. Genome Biol. 15, 550 (2014).

36. Vissers, L. E. L. M. et al. A de novo paradigm for mental retardation. Nat. Genet. 42, 1109-1112 (2010).

37. C Yuen, R. K. et al. Whole genome sequencing resource identifies 18 new candidate genes for autism spectrum disorder. Nat. Neurosci. 20, 602-611 (2017).

38. Landrum, M. J. et al. ClinVar: public archive of relationships among sequence variation and human phenotype. Nucleic Acids Res. 42, D980-5 (2014).

39. Dunham, I. et al. An integrated encyclopedia of DNA elements in the human genome. Nature 489, 57-74 (2012).

40. Miguel-Escalada, I., Pasquali, L. \& Ferrer, J. Transcriptional enhancers: Functional insights and role in human disease. Current Opinion in Genetics and Development 33, 71-76 (2015).

41. Osterwalder, M. et al. Enhancer redundancy provides phenotypic robustness 
in mammalian development. Nature 554, 239-243 (2018).

42. di lulio, J. et al. The human noncoding genome defined by genetic diversity. Nat. Genet. 50, 333-337 (2018).

43. Won, $\mathrm{H}$. et al. Chromosome conformation elucidates regulatory relationships in developing human brain. Nature 538, 523-527 (2016).

44. Whalen, S., Truty, R. M. \& Pollard, K. S. Enhancer-promoter interactions are encoded by complex genomic signatures on looping chromatin. Nat. Genet. 48, 488-496 (2016).

45. Vissers, L. E. L. M., Gilissen, C. \& Veltman, J. A. Genetic studies in intellectual disability and related disorders. Nature Reviews Genetics 17, 9-18 (2016).

46. Abrahams, B. S. et al. SFARI Gene 2.0: a community-driven knowledgebase for the autism spectrum disorders (ASDs). Mol. Autism 4, 36 (2013).

47. Lek, M. et al. Analysis of protein-coding genetic variation in 60,706 humans. Nature 536, 285-291 (2016).

48. Andersen, B. \& Rosenfeld, M. G. POU Domain Factors in the Neuroendocrine System: Lessons from Developmental Biology Provide Insights into Human Disease*. Endocr. Rev. 22, 2-35 (2001).

49. Nakaya, N., Sultana, A., Lee, H.-S. \& Tomarev, S. I. Olfactomedin 1 interacts with the Nogo A receptor complex to regulate axon growth. J. Biol. Chem. 287, 37171-84 (2012).

50. Kraus, D. M. et al. CSMD1 is a novel multiple domain complement-regulatory protein highly expressed in the central nervous system and epithelial tissues. J. Immunol. 176, 4419-30 (2006).

51. Dheedene, A., Maes, M., Vergult, S. \& Menten, B. A de novo POU3F3 Deletion in a Boy with Intellectual Disability and Dysmorphic Features. Mol. Syndromol. 5, 32-5 (2013).

52. Snijders Blok, L. et al. De Novo Variants Disturbing the Transactivation Capacity of POU3F3 Cause a Characteristic Neurodevelopmental Disorder. Am. J. Hum. Genet. 105, 403-412 (2019).

53. Zhao, J., Li, D., Seo, J., Allen, A. S. \& Gordân, R. Quantifying the Impact of Non-coding Variants on Transcription Factor-DNA Binding. Res. Comput. Mol. Biol. ... Annu. Int. Conf. RECOMB ... proceedings. RECOMB (Conference 2005-) 10229, 336-352 (2017). 
54. Molenaar, J. J. et al. Sequencing of neuroblastoma identifies chromothripsis and defects in neuritogenesis genes. Nature 483, 589-593 (2012).

55. Ripke, S. et al. Genome-wide association study identifies five new schizophrenia loci. Nat. Genet. 43, 969-976 (2011).

56. Kim, C.-H. et al. Repressor activity of Headless/Tcf3 is essential for vertebrate head formation. Nature 407, 913-916 (2000).

57. Ramakrishnan, A.-B. \& Cadigan, K. M. Wnt target genes and where to find them. F1000Research 6, (2017).

58. Gribble, S. L., Kim, H.-S., Bonner, J., Wang, X. \& Dorsky, R. I. Tcf3 inhibits spinal cord neurogenesis by regulating sox4a expression. Development 136, 781-789 (2009).

59. Groner, A. C. et al. KRAB-Zinc Finger Proteins and KAP1 Can Mediate LongRange Transcriptional Repression through Heterochromatin Spreading. PLoS Genet. 6, e1000869 (2010).

60. Gilbert, L. A. et al. CRISPR-mediated modular RNA-guided regulation of transcription in eukaryotes. Cell 154, 442-51 (2013).

61. O'Geen, H. et al. dCas9-based epigenome editing suggests acquisition of histone methylation is not sufficient for target gene repression. Nucleic Acids Res. 45, 9901-9916 (2017).

62. Lonsdale, J. et al. The Genotype-Tissue Expression (GTEx) project. Nat. Genet. 45, 580-585 (2013).

63. Schiaffino, S., Rossi, A. C., Smerdu, V., Leinwand, L. A. \& Reggiani, C. Developmental myosins: expression patterns and functional significance. Skelet. Muscle 5, 22 (2015).

64. Molumby, M. J., Keeler, A. B. \& Weiner, J. A. Homophilic Protocadherin CellCell Interactions Promote Dendrite Complexity. Cell Rep. 15, 1037-1050 (2016).

65. Coll-Tané, M., Krebbers, A., Castells-Nobau, A., Zweier, C. \& Schenck, A. Intellectual disability and autism spectrum disorders 'on the fly': insights from Drosophila. Dis. Model. Mech. 12, (2019).

66. Srivastava, A. K. \& Schwartz, C. E. Intellectual disability and autism spectrum disorders: causal genes and molecular mechanisms. Neurosci. Biobehav.

Rev. 46 Pt 2, 161-74 (2014). 
67. Werling, D. M. et al. An analytical framework for whole-genome sequence association studies and its implications for autism spectrum disorder. Nat. Genet. 50, 727-736 (2018).

68. Villar, D. et al. Enhancer Evolution across 20 Mammalian Species. Cell 160, 554-566 (2015).

69. Wilfert, A. B., Sulovari, A., Turner, T. N., Coe, B. P. \& Eichler, E. E. Recurrent de novo mutations in neurodevelopmental disorders: properties and clinical implications. Genome Med. 9, 101 (2017).

70. Stepanov, V., Marusin, A., Vagaitseva, K., Bocharova, A. \& Makeeva, O. Genetic Variants in CSMD1 Gene Are Associated with Cognitive Performance in Normal Elderly Population. Genet. Res. Int. 2017, 1-5 (2017).

71. Håvik, B. et al. The Complement Control-Related Genes CSMD1 and CSMD2 Associate to Schizophrenia. Biol. Psychiatry 70, 35-42 (2011).

72. Donohoe, G. et al. Neuropsychological effects of the CSMD1 genome-wide associated schizophrenia risk variant rs10503253. Genes, Brain Behav. 12, 203-209 (2013).

73. Steen, V. M. et al. Neuropsychological Deficits in Mice Depleted of the Schizophrenia Susceptibility Gene CSMD1. PLoS One 8, e79501 (2013). 
a

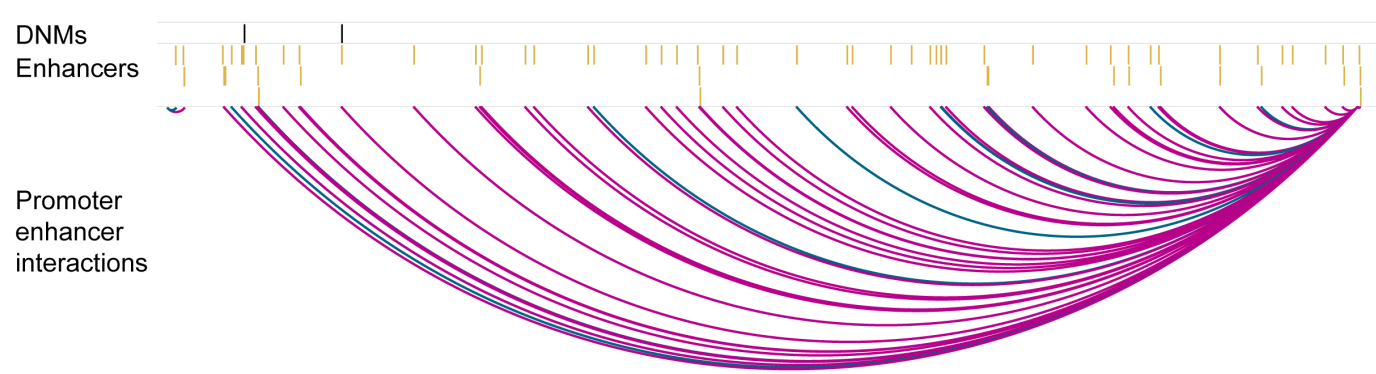

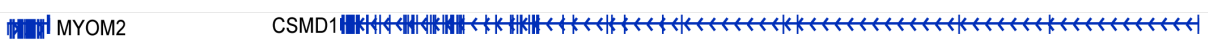

b

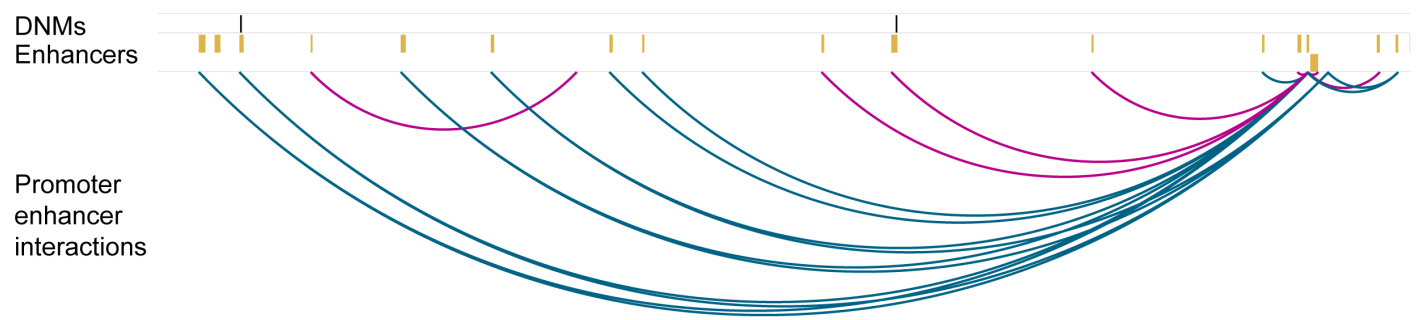

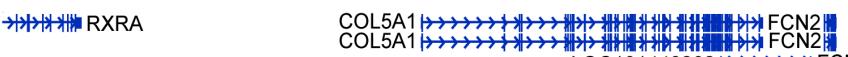

OLFM1 OC101448202世世FCN1

c

DNMs

Enhancers

Promoter

interactions

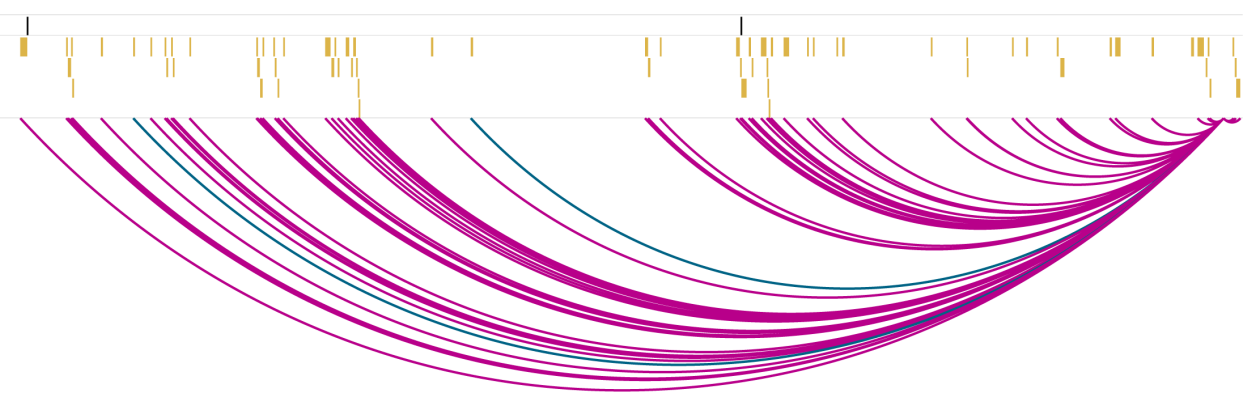

LOC100287010 HAH

LINC01114 POA POUF3|
LOC100506421

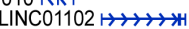

Figure S1: Recurrent de novo mutations (DNMs) in enhancer clusters: a)

Recurrent DNMs in CSMD1 enhancer cluster. b) Recurrent DNMs in OLFM1 enhancer cluster. c) Recurrent DNMs in POU3F3 enhancer cluster. Black lines indicate DNMs while yellow bars indicate enhancers. Pink arcs represent fetal brain specific enhancer-promoter interactions while green arcs represent human gain enhancer-promoter interactions. 